\title{
Dynamic Positioning Filter Method based on EnKF
}

\author{
Xiaogong Lin a , Ruxun Wang ${ }^{\mathrm{b}}$ and Dawei Zhao ${ }^{\mathrm{c}}$ \\ School of Automation, Harbin Engineering University, Harbin 150001, China. \\ alinxiaogong@hrbeu.edu.cn, b807147575@qq.com, cdaweizhao@hrbeu.edu.cn
}

Keywords: Dynamic positioning, nonlinear system state estimation, EnKF, Non-linear observer.

\begin{abstract}
In order to improve the positioning accuracy and reliability of the dynamic positioning system and deal with the problem of state estimation of nonlinear system with Gaussian noise, according to the basic principles and methods of Ensemble Kalman Filter (EnKF), a dynamic positioning filtering method is proposed based on EnKF. Then, the simulation results show that the nonlinear observer based on EnKF can effectively estimate the state of the ship and have certain robustness to the observed outliers. The validity of this method is verified at the end of this article.
\end{abstract}

\section{Introduction}

Dynamic positioning system is a kind of high-tech control technology, which is widely used in ships and offshore floating operation platforms. This system also plays a very important role in the development of marine resources [1]. In order to feedback the low-frequency signals to the controller, the state estimator of the ship need to filter out the noise of measurement, which is divided into two parts, low and high frequencies. According to kinematics and dynamics, a mathematical model of the ship can be built. Since the model is non-linear, the ship state estimation can be transformed into nonlinear filtering problem [2].

On the basis of measuring the observable signals of the system, filter theory can estimate the state of system according to certain estimation criteria. The most classical filtering theory is Kalman filter, but the traditional Kalman filter is only applicable to the state equation and the measurement equations which is fitting to the random linear Gaussian system, not to nonlinear systems.

Nonlinear filtering has been widely used in statistical signal processing, target tracking, satellite navigation and so on [3]. The key to the optimal filtering in nonlinear systems is to get the posterior probability density function exactly in system state. The representative methods in the nonlinear filtering are Extended Kalman Filter (EKF) [4], Unscented Kalman Filter (UKF) [5], Cubature Kalman Filter (CKF) [6] and so on. The most traditional method which is widely used in nonlinear Gaussian filtering is Extended Kalman Filter (EKF).By linearly truncating the nonlinear state and Taylor series expansion of the measurement function, the nonlinear system is transformed into a linear Kalman filtering system, so EKF is a sub-optimal filter. The main idea of this filter is to use the Non-linear in the parametric analytical systems to approximate. So the filtering accuracy is not good enough to be accepted. Long-term engineering application practice also shows that EKF is easy to divergence and has bad robustness when existing uncertainties in the system model.

Everson published the Ensemble Kalman Filter at the end of last century. EnKF initially aims to deal with the problem of data assimilation, and its principle combines the idea of the predictions of set on the basis of Kalman filter, which resulted in the convenient use of filtering algorithm, and promoted the vigorous development of filtering research.

Based on the theory of EnKF, the design of dynamic positioning observer is proposed under the unknown time-varying condition for Gaussian noise statistics. The nonlinear observer based on EnKF is used in dynamic positioning system, and the simulation results verify its effectiveness. 


\section{Ensemble Kalman Filter Principle}

\subsection{Optimal Bayesian Estimation.}

Consider the following nonlinear state space model:

$$
\begin{gathered}
x_{k}=f_{k}\left(x_{k-1}, w_{k-1}\right) \\
z_{k}=h_{k}\left(x_{k}, v_{k}\right)
\end{gathered}
$$

Here $x_{k} \in R^{n}$ is system state vector and $z_{k} \in R^{m}$ is observe vector, $f_{k-1}(\cdot)$ and $h_{k}(\cdot)$ respectively describe the non-linear state transition function and observation function. At the same time, the dynamic process noise $w_{k} \in R^{n}$ and the measurement noise $v_{k} \in R^{m}$ are also incorporated into the model. If the initial probability density function of the state is $p\left(x_{0} \mid z_{0}\right)=p\left(x_{0}\right)$, then the prediction and update process are as follows:

$$
\begin{gathered}
p\left(x_{k} \mid Z^{k-1}\right)=\int p\left(x_{k}, x_{k-1}\right) p\left(x_{k-1} \mid Z^{k-1}\right) d x_{k-1} \\
p\left(x_{k} \mid Z^{k}\right)=\frac{p\left(z_{k} \mid x_{k}\right) p\left(x_{k} \mid Z^{k-1}\right)}{\int p\left(z_{k} \mid x_{k}\right) p\left(x_{k} \mid Z^{k-1}\right) d x_{k}}
\end{gathered}
$$

Where $Z^{k}=\left\{z_{1}, z_{2}, \cdots, z_{k}\right\}$ describe the measurements from discrete time 1 to k. Equation (3) and (4) describe the basic idea of optimal Bayesian estimation. However, the integral in Equation (3) can only be obtained for some linear dynamic systems. For non-Gaussian nonlinear systems, how to obtain accurate numerical solutions has always been a research direction.

\subsection{Extended Kalman Filter.}

Let the initial state $x_{0}$ be independent of $w_{k}$ and $v_{k}$, the mean and covariance of $x_{0}$ are:

$$
\begin{gathered}
\widehat{\mathrm{x}}_{0}=E\left(x_{0}\right) \\
P_{0}=\operatorname{Cov}\left(x_{0}, x_{0}\right)=E\left[\left(x_{0}-\widehat{\mathrm{x}}_{0}\right)\left(x_{0}-\widehat{\mathrm{x}}_{0}\right)^{T}\right]
\end{gathered}
$$

The non-linear state function $f_{k-1}($.$) is done by Taylor deployment when \widehat{x}_{k-1}$ is decided by $k-1$. Then the higher order term of the second order is omitted, and the basic linear Kalman filter equation can be used to derive EKF as follows:

The step of State prediction:

$$
\widehat{\mathrm{X}}_{k \mid k-1}=\Phi_{k \mid k-1} \widehat{\mathrm{X}}_{k-1}+U_{k-1}
$$

The step of measure prediction:

$$
\widehat{\mathrm{z}}_{k \mid k-1}=H_{k} \widehat{\mathrm{x}}_{k \mid k-1}+y_{k}
$$

Covariance prediction:

$$
P_{k \mid k-1}=\Phi_{k \mid k-1} P_{k-1} \Phi_{k, k-1}^{T}+\Gamma_{k, k-1} Q_{k-1} \Gamma_{k, k-1}^{T}
$$

State estimation:

$$
\widehat{\mathrm{X}}_{k}=\widehat{\mathrm{X}}_{k \mid k-1}+K_{k}\left(\mathrm{z}_{k}-\widehat{z}_{k \mid k-1}\right)
$$

Filter gain:

$$
K_{k}=P_{k \mid k-1} H_{k}^{T}\left(H_{k} P_{k \mid k-1} H_{k}^{T}+\Lambda_{k} R_{k} \Lambda_{k}^{T}\right)^{-1}
$$

Covariance update:

$$
P_{k}=\left(I-K_{k} H_{k}\right) P_{k \mid k-1}
$$

Although EKF is widely used in engineering, it has many shortcomings and limitations. In the model recursion, it is necessary to calculate the Jacobian matrix, resulting in difficulties of achieving the method. The accuracy of the system noise is reduced when the noise is not Gaussian. It is not suitable for the strong nonlinearity, which requires the system state space to be continuous. These problems will lead to poor filtering stability and even divergence [7].

\subsection{Ensemble Kalman Filter.}

The Ensemble Kalman filter is developed on the basis of Bayesian filtering. Its core is to estimate the covariance between the state vector and the observation vector according to the prediction result 
of the set (finite sample), and then use the observation information and covariance information to analyze and update, the calculated result set is recursively forwarded to predict.

Assuming that set $X$ is the state of the system and this set is a state matrix consisting of $N$ sets of members, each member is consisting of $n$ states, expressed as:

$$
X_{k}=\left[x_{k}^{1}, x_{k}^{2}, x_{k}^{3}, \cdots, x_{k}^{i}, \cdots, x_{k}^{N},\right]
$$

Where $X_{k} \in R^{n \times N}$ represents the set of states at time $k, \quad x_{k}^{i} \in R^{n \times 1}$ represents the i-th state vector. Assume that the system is observed as $z \in R^{m}, Z$ is an observation set consisting of $N$ perturbed observation vectors:

$$
\begin{gathered}
z_{k}^{i}=z_{k}+\varepsilon_{k}^{i} \\
Z_{k}=\left[z_{k}^{1}, z_{k}^{2}, z_{k}^{3}, \cdots, z_{k}^{i}, \cdots z_{k}^{N}\right] \\
\gamma_{k}=\left[\varepsilon_{k}^{1}, \varepsilon_{k}^{2}, \varepsilon_{k}^{3}, \cdots, \varepsilon_{k}^{i}, \cdots \varepsilon_{k}^{N}\right]
\end{gathered}
$$

Where $Z_{k} \in R^{m \times N}$ is the observation matrix at time $k, z_{k}^{i} \in R^{m \times 1}$ is the observation vector of the i-th set at time $k, \varepsilon_{k}^{i} \in R^{m \times 1}$ is the perturbation vector of the i-th set at time $k, \gamma_{k} \in R^{m \times N}$ represents the perturbation matrix at time $k$.

The prediction set at the next time is obtained by the state transition function $\left\{x_{i}^{f}, i=1,2, \ldots N\right\}$, expressed as:

$$
x_{i}^{f}=f\left(x_{k}^{i}\right)
$$

Where $x_{i}^{f}$ is the predicted state of the i-th particle, and $f(\cdot)$ is the state transition function.

The Ensemble Kalman filter, like other Kalman filters, assumes that the prior distribution is represented by a Gaussian function whose mean and variance are as follows:

$$
\begin{gathered}
\overline{x_{k}^{f}}=\frac{1}{N} \sum_{i=1}^{N} x_{k, i}^{f} \\
P_{k}^{f}=\frac{1}{N-1} \sum_{i=1}^{N}\left(x_{k, i}^{f}-\overline{x_{k}^{f}}\right)\left(x_{k, i}^{f}-\overline{x_{k}^{f}}\right)^{T}
\end{gathered}
$$

Where $f$ is the prediction of the members of the particle set, $\overline{x_{k}^{f}}$ represents the predicted mean at time $k, P_{k}^{f}$ represents the predicted variance at time $k$, The parameter $x_{k, i}^{f}$ represents the predicted state of the i-th particle at time $k$, When $N$ tends to infinity, the mean and variance of the prediction state matrix will reach the true value of mean and variance.

During the update phase, each of the EnKF members uses the Kalman filter principle:

$$
x_{i}^{a}=x_{i}^{f}+K\left[z_{i}-h\left(x_{i}^{f}\right)\right]
$$

The measurement function is non-linear as shown in the following equation:

$$
z_{k}=h\left(x_{k}, v_{k}\right)
$$

$\overline{P^{f}} H^{T}$ and $H \overline{P^{f}} H^{T}$ are defined as follows:

$$
\begin{gathered}
P^{f} H^{T}=\frac{1}{N-1} \sum_{i=1}^{N}\left[x_{i}^{f}-\overline{x^{f}}\right]\left[h\left(x_{i}^{f}\right)-\overline{h\left(x^{f}\right)}\right]^{T} \\
H P^{f} H^{T}=\frac{1}{N-1} \sum_{i=1}^{N}\left[h\left(x_{i}^{f}\right)-\overline{h\left(x^{f}\right)}\right]\left[h\left(x_{i}^{f}\right)-\overline{h\left(x^{f}\right)}\right]^{T}
\end{gathered}
$$

Where

$$
\overline{h\left(x^{f}\right)}=\frac{1}{N} \sum_{i=1}^{N} h\left(x_{i}^{f}\right)
$$

The Kalman gain for a nonlinear system can be written as follows:

$$
K=P_{x z} P_{z z}^{-1}
$$


Where $P_{x z}$ is the crossed covariance of the state error and the observed error, $P_{z z}$ is the error covariance of the observed value and the predicted value of $h\left(x^{f}\right)$.

The error covariance matrix of equation (21) can be calculated as follows:

$$
\begin{gathered}
P_{x z}=\frac{1}{N-1} \sum_{i=1}^{N}\left[x_{i}^{f}-\overline{x^{f}}\right]\left[h\left(x_{i}^{f}\right)-h\left(\overline{x^{f}}\right)\right]^{T} \\
P_{z z}=\frac{1}{N-1} \sum_{i=1}^{N}\left[h\left(x_{i}^{f}\right)-h\left(\overline{x^{f}}\right)\right]\left[h\left(x_{i}^{f}\right)-h\left(\overline{x^{f}}\right)\right]^{T}+R
\end{gathered}
$$

Summarize the main equation of EnKF as follows:

$$
\begin{gathered}
x_{i}^{a}=x_{i}^{f}+K\left[y_{i}-h\left(x_{i}^{f}\right)\right] \\
K=\left\{\frac{1}{N-1} \sum_{i=1}^{N}\left[x_{i}^{f}-\overline{x^{f}}\right]\left[h\left(x_{i}^{f}\right)-h\left(\overline{x^{f}}\right)\right]^{T}\right\} *\left\{\frac{1}{N-1} \sum_{i=1}^{N}\left[h\left(x_{i}^{f}\right)-h\left(\overline{x^{f}}\right)\right]\left[h\left(x_{i}^{f}\right)-h\left(\overline{x^{f}}\right)\right]^{T}+R\right\}^{-1}
\end{gathered}
$$

Equation (29) is the improved Kalman gain.

Compared with the traditional method, this method reduces the computational complexity and makes a better assimilation analysis of the nonlinear system measurement function. Using the idea of ensemble prediction to avoid the solution of complex error covariance, thus slow down the computational pressure, and solve the shortcomings of calculating EKF difficultly.

\section{Nonlinear Observer Design}

\subsection{Mathematical Modeling of Ships}

In this paper, we utilize low speed dynamic positioning vessel as the research object, ignoring roll, pitch and heave motion, only considering horizontal movement of the ship, and then three degrees of freedom motion of high frequency and low frequency model motion model were established respectively.

Due to attitude and line speed vessel were expressed in the north-east coordinate system and the ship coordinate system, so we take advantage of the Euler transform to do its conversion, the conversion relationship as follows[8,9]:

$$
\dot{\boldsymbol{\eta}}=R(\psi) \mathbf{v}
$$

Where $\boldsymbol{R}(\psi)=\left[\begin{array}{ccc}\cos \psi & -\sin \psi & 0 \\ \sin \psi & \cos \psi & 0 \\ 0 & 0 & 1\end{array}\right]$ is a state-dependent transformation matrix. Notice that $\boldsymbol{R}^{\mathrm{T}}(\psi)=\boldsymbol{R}^{-1}(\psi)$. Let the NE position $(x, y)$ and heading $\psi$ of the vessel relative to a $\mathrm{NE}$ frame $O_{E}-X_{E} Y_{E} Z_{E}$ be expressed in vector form by $\boldsymbol{\eta}=[x, y, \psi]^{\mathrm{T}}$, and let the velocities decomposed in a hull reference frame be represented by the state vector $v=[u, v, r]^{\mathrm{T}}$.

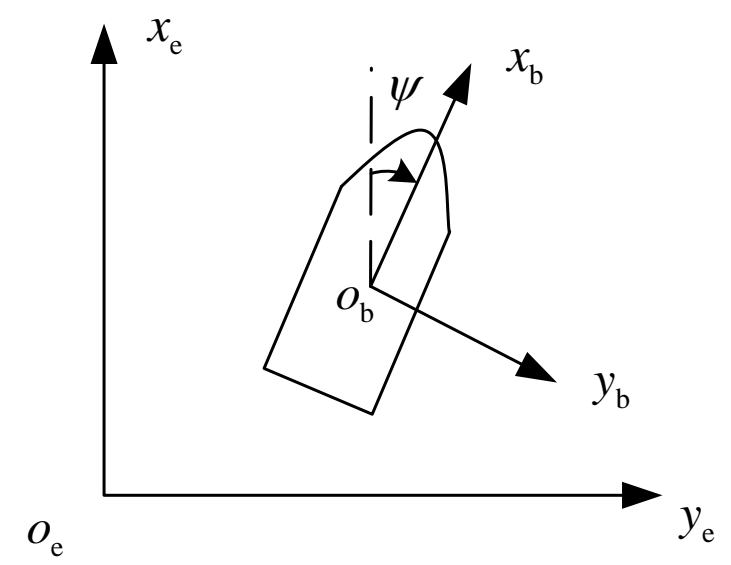

Fig.1 ship’s movement in the horizontal plane 
For a large class of surface vessel, we can use following mathematical model to describe the low-frequency motion.

$$
\begin{gathered}
\mathbf{M} \dot{\boldsymbol{v}}+\mathbf{D} \boldsymbol{v}=\boldsymbol{\tau}+\mathbf{R}^{\mathrm{T}}(\psi) \mathbf{b} \\
\boldsymbol{\tau}=\mathbf{B}_{u} \mathbf{u} \\
\dot{\boldsymbol{b}}=-\boldsymbol{T}^{-1} \boldsymbol{b}+\boldsymbol{E}_{\mathrm{b}} \boldsymbol{\omega}_{\mathrm{b}}
\end{gathered}
$$

Where $\boldsymbol{\tau}=\left[\begin{array}{lll}X & Y & Z\end{array}\right]^{\mathrm{T}}$ denote the propeller thrust, $\mathbf{b} \in \mathbf{R}^{3}$ is a vector of bias forces and moments on the supposition that the forces due to wind wave and drift are slowly varying. $\mathbf{B}_{u} \in \mathbf{R}^{3 \times r}$ is a constant matrix which represents the transmission between the input and the thrust. $\mathbf{u} \in \mathbf{R}^{r}(r \geq 3)$ is the input, $\boldsymbol{T}=\operatorname{diag}\left\{T_{11}, T_{22}, T_{33}\right\}$ is a diagonal matrix of positive bias time constants and $\boldsymbol{E}_{b}=\operatorname{diag}\left\{\sigma_{4}, \sigma_{5}, \sigma_{6}\right\}$ is a diagonal matrix scaling the amplitude of the environment disturbance. If a small Froude number is assumed, the inertia matrix $\boldsymbol{M}=\boldsymbol{M}^{\mathrm{T}}>0$ which includes hydrodynamic added inertia can be written:

$$
\mathbf{M}=\left[\begin{array}{ccc}
m-X_{\dot{u}} & 0 & 0 \\
0 & m-Y_{\dot{v}} & m x_{G}-Y_{\dot{r}} \\
0 & m x_{G}-Y_{\dot{r}} & I_{z}-N_{\dot{r}}
\end{array}\right]
$$

For a straight-line stable ship, $\boldsymbol{D}$ will be a strictly positive damping matrix due to linear wave drift damping and laminar skin friction. The linear damping matrix is defined as:

$$
\mathbf{D}=\left[\begin{array}{ccc}
-X_{u} & 0 & 0 \\
0 & -Y_{v} & -Y_{r} \\
0 & -N_{v} & -N_{r}
\end{array}\right]
$$

Where $m$ is the vessel mass and $I_{z}$ is the moment of inertia about the vessel-fixed z-axis. $x_{G}$ is the center of gravity about the vessel-fixed z-axis. The zero-frequency added mass in surge, sway and yaw due to accelerations along the corresponding axes are defined as $X_{\dot{u}}<0, Y_{\dot{v}}<0$ and $N_{\dot{r}}<0, Y_{\dot{r}}$ is the added mass coupled in sway and yaw and is also defined as negative.

In this article, the second order model is used to describe the ship motion caused by first order wave force, the state space modal is shown below.

$$
\begin{gathered}
{\left[\begin{array}{l}
\dot{\boldsymbol{\xi}}_{1} \\
\dot{\boldsymbol{\xi}}_{2}
\end{array}\right]=\left[\begin{array}{cc}
\mathbf{0} & \mathbf{I} \\
\boldsymbol{\Omega}_{21} & \boldsymbol{\Omega}_{22}
\end{array}\right]\left[\begin{array}{l}
\boldsymbol{\xi}_{1} \\
\boldsymbol{\xi}_{2}
\end{array}\right]+\left[\begin{array}{c}
\mathbf{0} \\
\mathbf{E}_{h 2}
\end{array}\right] \boldsymbol{\omega}_{h}} \\
\boldsymbol{\eta}_{h}=\left[\begin{array}{ll}
\mathbf{0} & \mathbf{I}
\end{array}\right]\left[\begin{array}{l}
\boldsymbol{\xi}_{1} \\
\boldsymbol{\xi}_{2}
\end{array}\right]
\end{gathered}
$$

Where $\boldsymbol{\xi}_{1} \in \boldsymbol{R}^{3}$ and $\boldsymbol{\xi}_{2} \in \boldsymbol{R}^{3}$ denote the WF state vector, $\boldsymbol{\Omega}_{21}=-\operatorname{diag}\left\{\omega_{01}^{2}, \omega_{02}^{2}, \omega_{03}^{2}\right\}$, $\boldsymbol{\Omega}_{22}=-\operatorname{dia}\left\{2 \zeta_{1} \omega_{01}, 2 \zeta_{2} \omega_{02}, 2 \zeta_{3} \omega_{03}\right\}, \boldsymbol{E}_{h 2}=\operatorname{diag}\left\{\sigma_{1}, \sigma_{2}, \sigma_{3}\right\}$.

\subsection{EnKF Observer Design}

In this paper, the main task is to design a Nonlinear Observer for Ships Based on Ensemble Kalman Filter which can filter out high frequency motion so that the low frequency motion feedback to the control system owing to Dynamic Positioning measuring system usually only get the vessel's position and heading information.

Observation equation as follows:

$$
\begin{gathered}
\mathrm{d} x=\mathbf{f}(\mathbf{x}) \mathrm{d} t+\mathbf{B u d} t+\mathbf{E d} \boldsymbol{\beta} \\
\mathbf{y}_{k}=\mathbf{H} \mathbf{x}_{k}+\mathbf{v}_{k}, \quad k=1,2,3 \cdots
\end{gathered}
$$

Where $\boldsymbol{x}=\left[\boldsymbol{\xi}^{\mathrm{T}}, \boldsymbol{\eta}^{\mathrm{T}}, \boldsymbol{b}^{\mathrm{T}}, \boldsymbol{v}^{\mathrm{T}}\right] \in \boldsymbol{R}^{15}$ is state vector and $\boldsymbol{H}=\left[\begin{array}{llll}\boldsymbol{\Gamma} & \boldsymbol{I}_{3 \times 3} & \boldsymbol{0}_{3 \times 3} & \boldsymbol{0}_{3 \times 3}\end{array}\right]$. The desired north position, east position and heading angle can be written as $\boldsymbol{u}=\left[\begin{array}{lll}x_{r} & y_{r} & \psi_{r}\end{array}\right]^{\mathrm{T}} \in \boldsymbol{R}^{3}$ which is a controllable vector. $\boldsymbol{\beta} \in \boldsymbol{R}^{15}$ is standard Brownian motion, so d $\boldsymbol{\beta}$ is standard Gaussian process. $\boldsymbol{y}_{k} \in \boldsymbol{R}^{3}$ is the output vector which represents the DGPS and gyrocompass measurements polluted by the measured noise when the time is $t=k T$ and $\boldsymbol{x}_{k} \in \boldsymbol{R}^{3}$ is the state vector of the system. T is the measurement cycle of the DGPS and 
gyrocompass. The covariance matrix $\boldsymbol{R}_{k}$ is related to the accuracy of $\boldsymbol{v}_{k} \sim \mathrm{N}\left(\boldsymbol{0}, \boldsymbol{R}_{k}\right)$ which is the measurement noise vector. Equation vector $\boldsymbol{f}(\boldsymbol{x})$, input matrix $\boldsymbol{B}$ and process noise amplitude matrix $\boldsymbol{E}$ can be written:

$$
\begin{gathered}
\boldsymbol{f}(\boldsymbol{x})=\left[\begin{array}{c}
\boldsymbol{\Omega} \boldsymbol{\xi} \\
\boldsymbol{R}\left(\psi+\psi_{h}\right) \boldsymbol{v} \\
-\boldsymbol{T}^{-1} \boldsymbol{b} \\
\boldsymbol{M}^{-1} \boldsymbol{D} \boldsymbol{v}+\boldsymbol{M}^{-1} \boldsymbol{R}^{T}\left(\psi+\psi_{h}\right) \boldsymbol{b}
\end{array}\right] \\
\boldsymbol{B}=\left[\begin{array}{c}
\boldsymbol{0}_{6 \times p} \\
\boldsymbol{0}_{3 \times p} \\
\boldsymbol{0}_{3 \times p} \\
\boldsymbol{M}^{-1} \boldsymbol{B}_{u}
\end{array}\right] \\
\mathbf{E}=\left[\begin{array}{ccc}
\mathbf{E}_{\mathrm{h}} & \mathbf{0}_{6 \times 3} & \mathbf{0}_{6 \times 3} \\
\mathbf{0}_{3 \times 3} & \mathbf{0}_{3 \times 3} & \mathbf{0}_{3 \times 3} \\
\mathbf{0}_{3 \times 3} & \mathbf{E}_{\mathrm{b}} & \mathbf{0}_{3 \times 3} \\
\mathbf{0}_{3 \times 3} & \mathbf{0}_{3 \times 3} & \mathbf{E}_{\mathrm{s}}
\end{array}\right] \\
\mathbf{H}=\left[\begin{array}{llll}
\boldsymbol{\Gamma} & \mathbf{I}_{3 \times 3} & \mathbf{0}_{3 \times 3} & \mathbf{0}_{3 \times 3}
\end{array}\right]
\end{gathered}
$$

Considering the continuous-discrete system:

$$
\begin{gathered}
\mathrm{d} \mathbf{x}(t)=\mathbf{f}(\mathbf{x}(t), t) \mathrm{d} t+\sqrt{\mathbf{Q}} \mathrm{d} \boldsymbol{\beta}(t) \\
\mathbf{z}_{k}=\mathbf{h}\left(\mathbf{x}_{k}, k\right)+\mathbf{v}_{k}, \quad k=1,2,3, \cdots
\end{gathered}
$$

Where $\boldsymbol{x}(t) \in \boldsymbol{R}^{n}$ independent with $\mathrm{d} \beta(t)$ is the n-dimensional state vector of the system. $\boldsymbol{f}(\cdot)$ is the equation matrix with corresponding dimension. $\boldsymbol{Q} \in \boldsymbol{R}^{n \times n}$ is the gain matrix of process noise. $\boldsymbol{z}_{k} \in \boldsymbol{R}^{m}$ is the m-dimensional output vector, ie measurement vector at time step k. $\boldsymbol{E}\left[\boldsymbol{v}_{i} \boldsymbol{v}_{j}\right]=\boldsymbol{R}_{i} \delta_{i j}$ and $\boldsymbol{h}(\cdot)$ is the matrix with corresponding dimension.

Apply the numerical solution method composed by the Euler method and the Milstein method to introducing the discrete Kalman filter algorithm to the study of observer in continuous-discrete nonlinear system. So, disperse the system process equation (32) by using the 1.5-order Itô-Taylor method.

It is assumed that $\boldsymbol{x}(t)$ is known, then the approximate numerical solution at time $t+\delta$ can be written as:

$$
\mathbf{x}(t+\delta)=\underbrace{\mathbf{x}(t)+\delta \mathbf{f}(\mathbf{x}(t), t)+\frac{1}{2} \delta^{2}\left(\nabla_{0} \mathbf{f}(\mathbf{x}(t), t)\right)}_{\mathbf{f}_{d}(\mathbf{x}(t), t)}+\sqrt{\mathbf{Q}} \varepsilon+(\nabla \mathbf{f}(\mathbf{x}(t), t)) \mathbf{e}
$$

Where $\varepsilon$ and $\boldsymbol{e}$ are related Gaussian random vectors, they can be generated by two standard Gaussian random vector $\left(\boldsymbol{n}_{1}, \boldsymbol{n}_{2}\right)$ :

$$
\begin{gathered}
\boldsymbol{\varepsilon}=\sqrt{\delta} \mathbf{n}_{1} \\
\mathbf{e}=\frac{1}{2} \delta^{3 / 2}\left(\mathbf{n}_{1}+\frac{\mathbf{n}_{2}}{\sqrt{2}}\right)
\end{gathered}
$$

Hence, the covariance matrix can be written as:

$$
\begin{gathered}
\mathbf{E}\left[\boldsymbol{\varepsilon} \boldsymbol{\varepsilon}^{\mathrm{T}}\right]=\delta \mathbf{I}_{n} \\
\mathbf{E}\left[\boldsymbol{\varepsilon} e^{\mathrm{T}}\right]=\frac{1}{2} \delta^{2} \mathbf{I}_{n} \\
\mathbf{E}\left[e e^{\mathrm{T}}\right]=\frac{1}{3} \delta^{3} \mathbf{I}_{n}
\end{gathered}
$$

Besides, the two differential operator, $\nabla_{0}$ and $\nabla$ are defined as: 


$$
\begin{gathered}
\nabla_{0}=\frac{\partial}{\partial t}+\sum_{i=1}^{n} \mathbf{f}_{i} \frac{\partial}{\partial \mathbf{x}_{i}}+\frac{1}{2} \sum_{j, p, q=1}^{n} \sqrt{\mathbf{Q}_{p, j}} \sqrt{\mathbf{Q}_{j, p}} \mathbf{f}_{i} \frac{\partial^{2}}{\partial \mathbf{x}_{p} \partial \mathbf{x}_{q}} \\
\nabla_{j}=\sum_{i=1}^{n} \sqrt{\mathbf{Q}_{i j}} \frac{\partial}{\partial \mathbf{x}_{i}}
\end{gathered}
$$

$\nabla \boldsymbol{f}$ is square matrix, its element at $\operatorname{position}(i, j)$ is $\nabla_{j} f_{i}(i, j=1,2,3, \cdots, n)$.

At discrete time, the process equation to remove the noise is expressed as:

$$
\mathbf{f}_{d}(\mathbf{x}(t), t)=\mathbf{x}(t)+\delta f(\mathbf{x}(t), t)+\frac{1}{2} \delta^{2}\left(\nabla_{0} \mathbf{f}(x(t), t)\right)
$$

Based on the above discussion, we can transform the continuous model to discrete form. Now that we can design ship observer with EnKF state estimation algorithm, and the algorithm process was mentioned above.

\section{Simulation Results and Analysis}

\begin{tabular}{|c|c|c|c|}
\hline parameters & values & parameters & values \\
\hline length/m & 175 & $\begin{array}{l}\text { rolling radius of } \\
\text { gyration } / \mathrm{m}\end{array}$ & 8.3310 \\
\hline width/m & 25.4 & $\begin{array}{l}\text { pitching radius } \\
\text { of gyration /m }\end{array}$ & 42.000 \\
\hline draught/m & 9.5 & $\begin{array}{l}\text { yawing radius } \\
\text { of gyration /m }\end{array}$ & 42.000 \\
\hline $\begin{array}{c}\text { quality } \\
\text { of hull /kg }\end{array}$ & $\begin{array}{c}24, \\
609,620\end{array}$ & $\begin{array}{l}\text { center of } \\
\text { flotage/m }\end{array}$ & $\begin{array}{c}(-2.5470,0, \\
5.2070)\end{array}$ \\
\hline drainage /m3 & $\begin{array}{l}24 . \\
009\end{array}$ & $\begin{array}{l}\text { center of } \\
\text { gravity/m }\end{array}$ & $\begin{array}{c}(-2.5475,0, \\
9.5500)\end{array}$ \\
\hline $\begin{array}{c}\text { coefficient of } \\
\text { square }\end{array}$ & 0.5690 & $\begin{array}{l}\text { wetted surface } \\
\text { area/m2 }\end{array}$ & 4927.6 \\
\hline
\end{tabular}

A dynamic positioning ship parameters for the simulation are shown in table 1.

Table 1. Part of one DP vessel's hull parameters

Choose the mathematical model of the ship LF motion as (31), the parameters are:

$$
\begin{aligned}
& \mathbf{M}=\left[\begin{array}{ccc}
2.641532 \times 10^{7} & 0 & 0 \\
0 & 3.345547 \times 10^{7} & 1.491735 \times 10^{7} \\
0 & 1.491735 \times 10^{7} & 6.520948 \times 10^{10}
\end{array}\right] \\
& \mathbf{D}=\left[\begin{array}{ccc}
2.220421 \times 10^{4} & 0 & 0 \\
0 & 2.220421 \times 10^{5} & -1.774611 \times 10^{6} \\
0 & -1.774611 \times 10^{6} & 7.150578 \times 10^{8}
\end{array}\right]
\end{aligned}
$$

The time constant matrix $\boldsymbol{T}$ in (33) is:

$$
\mathbf{T}=\left[\begin{array}{ccc}
2500 & 0 & 0 \\
0 & 2500 & 0 \\
0 & 0 & 2500
\end{array}\right]
$$

Choose the mathematical model of the ship WF motion as (36). The relative damping factor is $\zeta_{\mathrm{i}}=0.15$, the dominant frequency of the wave spectrum is $\omega_{0 i}=0.9$. Sea state is 3 . The height of significant wave is $0.5 \sim 1.25 \mathrm{~m}$.

\subsection{Simulation with Gaussian White Noise}

In order to verify the designed observer's performance, select CKF observer for comparison, the simulation time is $200 \mathrm{~s}$, the simulation results are shown in figure 3 to 5. 


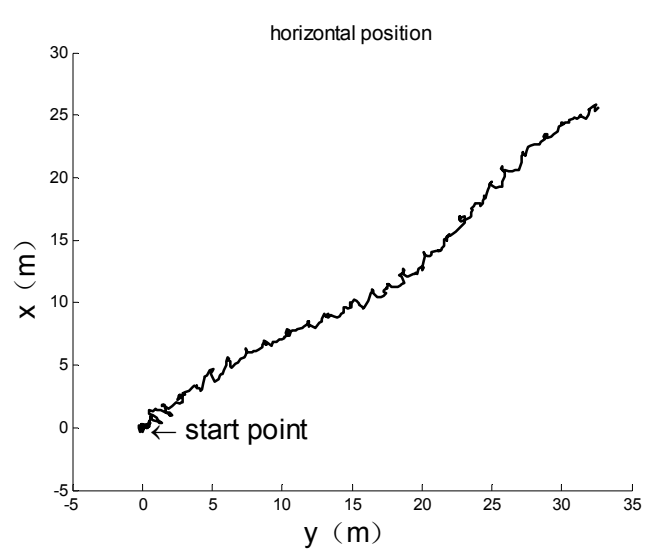

Fig.2 Horizontal position of vessel
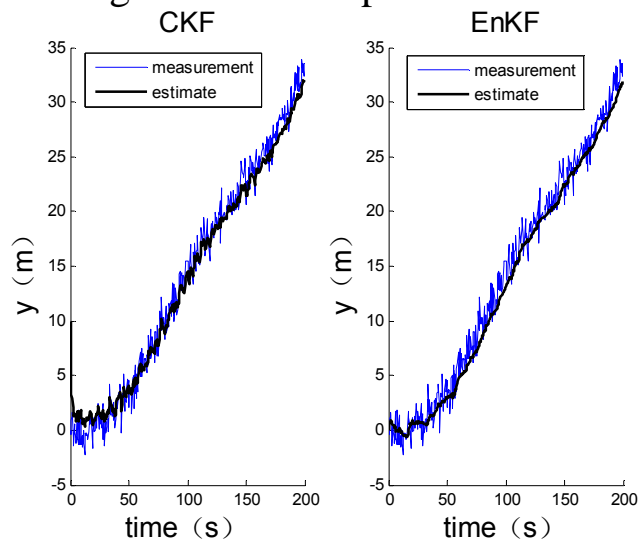

Fig.4 Ship sway displacement
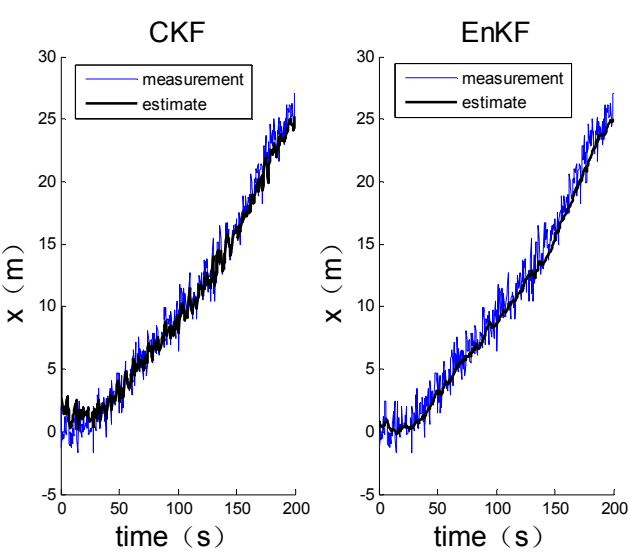

Fig.3 Ship surge displacement
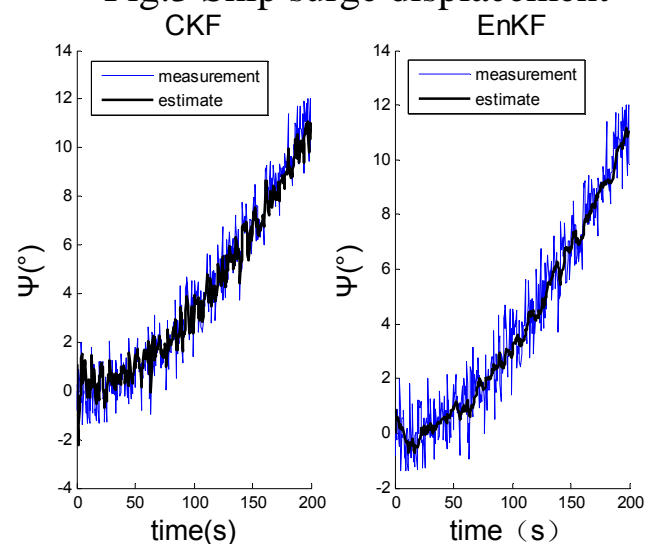

Fig.5 Ship yaw angle

\subsection{Simulation with Gaussian White Noise Add Outliers}

Add the outliers in measurements on 50s, other parameters keep the same value as above, and the simulation results are shown in figure 6 to 8 .

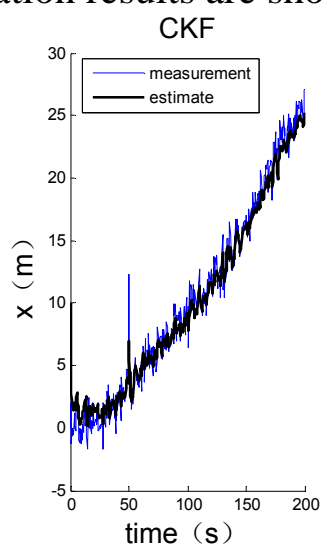

Fig.6 Ship surge displacement
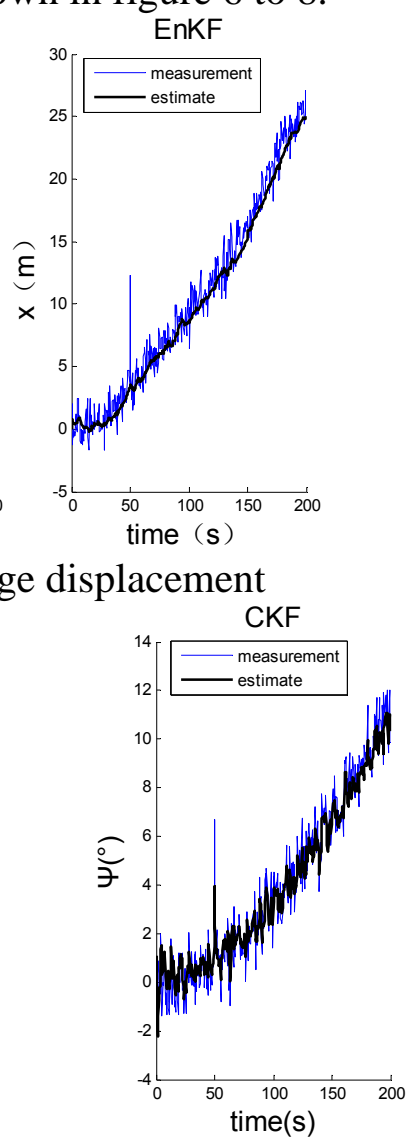
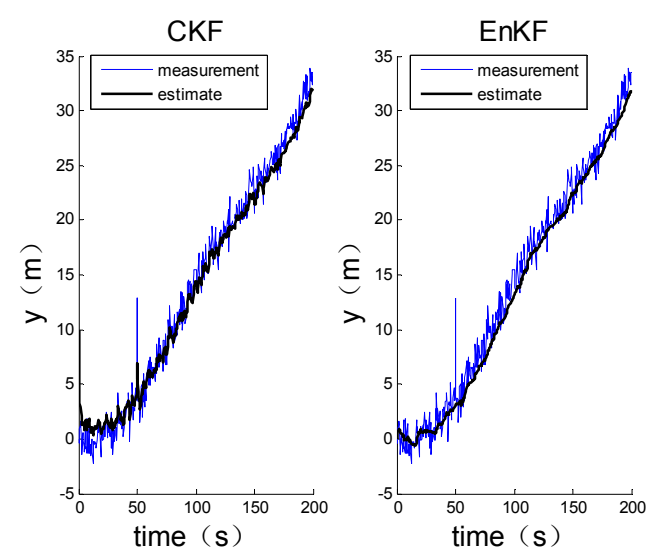

Fig.7 Ship sway displacement

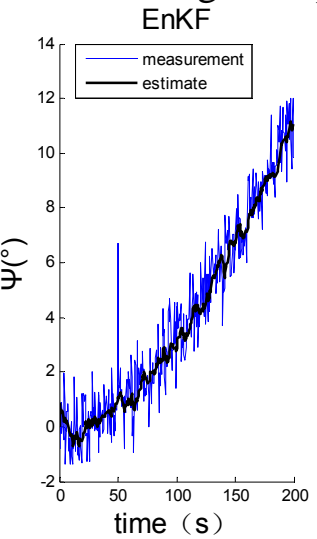

Fig.8 Ship yaw angle 


\section{Summary}

From the simulation results, EnKF observer and CKF observer proposed can estimate the position and heading of the ship in white noise environment, and the estimated curve after Ensemble Kalman filter is smoother, and bring better feedback for the controller.

In summary, the simulation results verify the validity of the EnKF observer. The disadvantage is that the non-Gaussian noise has not been modeled and simulated. This is for further study.

\section{References}

[1]. Zhou Li, Wang Lei, Chen Heng. Review on the Study of Dynamic Positioning Control System for Vessels. SHIP \& OCEAN ENGINEERING. Vol. 37 (2008) No. 2, p. 86-90.

[2]. Bian Xinqian, Fu Mingyu, Wang Yuanhui. Dynamic Positioning. Beijing: Science Press, 2011.

[3]. Baser, McDonald. A Joint Multitarget Estimator for the Joint Target Detection and Tracking Filter. IEEE Trans on Signal Processing, 2015.

[4]. S.Julier, J.Uhlmann. A New Extension of the Kalman Filter to Nonlinear Systems. Proceedings of AeroSence: The 11th International Symposium on Aerospace/Defense Sensing, Simulation and Controls, Orlando, Florida, 1997.

[5]. Van der Merwe R, Doucet A, Freitas N, Wan E A. The Unscented Particle Filter. Technical Report CUEDPF-INFENGPTR 380, Cambridge University, 2000.

[6]. Arasaratnam I, Haykon S. Cubature Kalman Filters. IEEE Transactions on Automatic Control, 2009, 54(6):1254-1269.

[7]. Zhu Lin. Research on Assembling Kalman Filter Assimilation Method. Nanjing University of Information Science \& Technology, 2007.

[8]. Li Dianpu. "Ship Motion and Modeling,” Harbin Engineering University Press. 1999:P1-P17

[9]. M.J. Morgan Dynamic positioning of offshore vessels, Beijing: National Defense Industry Press1984. 